\title{
Pronunciation of Hawaiian
}

\section{Consonants}

p, $\mathbf{k}$ about as in English but with less aspiration.

$\mathbf{h}, \mathbf{l}, \mathbf{m}, \mathbf{n}$ about as in English.

w after $i$ and $e$ usually like $v$; after $u$ and $o$ usually like $w$; initially and after $a$ like $v$ or $w$.

- a glottal stop, similar to the sound between the oh's in English oh-oh.

Vowels

Unstressed

a like $a$ in above

e like $e$ in bet

I like $y$ in city

- like $o$ in sole but without off-glides.

a like $o o$ in moon

Stressed

a, $\bar{a}$ like $a$ in far

e like $e$ in bet

$\overline{\mathbf{e}}$ like $a y$ in play

$\mathbf{i}, \overline{\mathbf{T}}$ like $e e$ in see

$\mathbf{0 ,} \overline{\mathbf{o}}$ like $o$ in sole $\begin{array}{ll}\mathbf{0}, \overline{\mathbf{u}} & \text { like } o o \text { in moon }\end{array}$ vowels.

\section{Diphthongs}

ei, en, oi, ou, ai, ae, ao, au

but without off-glides; vowels marked with macrons are somewhat longer than other

these are always stressed on the first member, but the two members are not as closely joined as in English.

\section{Stress (or Accent)}

On all vowels marked with macrons: $\bar{a}, \bar{e}, \boldsymbol{\imath}, \bar{o}, \bar{a}$.

Otherwise on the next-to-last syllable and alternating preceding syllables of words, except that words containing five syllables without macrons are stressed on the first and fourth syllables. Final stress in a word (') is usually louder than preceding stress or stresses ('): hále, makáu, hòlohólo, 'èlemakúle.

For more details see section 2 of the Grammar. 